\title{
Serum Zinc and Copper Levels in Gestational Diabetes Mellitus in a Tertiary Care Hospital of Bangladesh
}

\author{
Mishu FA ${ }^{\mathrm{a}}$ Muttalib MA ${ }^{\mathrm{b}}$, Sultana B
}

\begin{abstract}
Background: The term gestational diabetes mellitus (GDM) is becoming a major health problem in developing countries undergoing rapid changes in lifestyle, dietary habits and body mass index. GDM is associated with an increased incidence of congenital abnormalities which is also aggravated by mother's zinc and copper deficiency. Zinc and copper are essential trace elements for normal embryogenesis and fetal growth and their deficiency increase mortality and morbidity of mothers, embryos and neonates. This study was designed to evaluate the association of serum zinc and copper with GDM in second and third trimester.

Methods: It was a case-control study. This study was conducted in Mymensingh Medical College Hospital during the period from July 2013 to June 2014 to evaluate the association of zinc and copper levels of pregnant women with GDM. A total induded of 172 subjects were participated in this study; among them 86 women diagnosed with GDM were selected as case (Group-I) and 86 healthy pregnant women were control (GroupII).The case group was again subdivided as Group Ia and Ib according to second and third trimester respectively. Control group was also subdivided as Group II and IIb according to second and third trimester respectively. Student's unpaired 't'test was used to analyse the data between groups. For analytical purpose 95\% confidence limit $(p<0.05)$ was taken as level of significance.

Results: There was significant difference in serum zinc and copper levels in cases compared to control group. Highly significant difference $(p<0.001)$ was found when serum zinc was compared between women with GDM and normoglycemic pregnant women in second and third trimester. Serum copper level was significantly increased in cases compared to control group in second trimester and the difference was found highly significant $(p<0.001)$ and significant difference $(p<0.01)$ was found in GDM compared to normoglycemic pregnant women in third trimester.
\end{abstract}

Conclusion: Estimation of serum zinc and copper levels might be incorporated in every GDM cases for prevention of complications.

Keywords: Diabetes mellitus, Gestational Diabetes Mellitus, Oral Glucose Tolerance Test, Trace elements.

(BIRDEM Med J 2018; 8(1): 52-55)

\section{Author Information}

a. Dr. Farzana Akonjee Mishu, Senior Research Officer, Department of Physiology and Molecular Biology, Bangladesh Institute of Research \& Rehabilitation In Diabetes, Endocrine $\&$ Metabolic Disorders (BIRDEM) Dhaka, Bangladesh.

b. Dr. MA Muttalib, Professor of Biochemistry and Chief Scientific Officer, General Laboratory (Biochemistry, Hematology and Clinical Pathology),Bangladesh Institute of Research \& Rehabilitation In Diabetes, Endocrine \& Metabolic Disorders (BIRDEM) Dhaka, Bangladesh.

c. Dr. Bilkis Sultana, Scientific Assistant, Bangladesh Institute of Research \& Rehabilitation in Diabetes, Endocrine \& Metabolic Disorders (BIRDEM) Dhaka, Bangladesh.

Address of Correspondences: Dr. Farzana Akonjee Mishu, Senior Research Officer, Department of Physiology and Molecular Biology, Bangladesh Institute of Research \& Rehabilitation In Diabetes, Endocrine \& Metabolic Disorders (BIRDEM) Dhaka, Bangladesh. Email Address: farzanamishu@yahoo.co.uk

Receive : October 29, 2016

Accepted: October 31, 2017

\section{Introduction}

Gestational diabetes mellitus (GDM) is defined as glucose intolerance resulting in hyperglycemia, with first onset or detection during pregnancy. ${ }^{1,2}$ Usually initiation of GDM is in middle and late gestational period and continues to term ${ }^{3}$. Glucose intolerance usually returns to normal range within 6 weeks after delivery. ${ }^{4}$ Approximately $1-14 \%$ of all pregnancies are complicated by GDM. ${ }^{3}$ The incidence of GDM in Bangladesh is $6.7 \%$ among frequency of congenital malformation in infants of diabetic mothers is estimated to be $6-10 \% .^{5}$ Pregnancy is associated with physiological changes that result in increased plasma volume and red blood cells and decreased concentrations of plasma proteins and micronutrients. ${ }^{6}$ It is a time of increased nutritional needs, both to support 
the rapidly growing fetus and to allow for the changes occurring in the pregnant. ${ }^{7}$ Gestational diabetes is associated with excessive nutrient losses due to glycosuria. ${ }^{8}$ Different researchers demonstrated that micro and macro nutrients are essential for the development of fetus. Wang et al also showed zinc contents in serum of pregnant women with gestational diabetes decreased compared with normal pregnant women. ${ }^{9}$ With the progress of pregnancy, the level of plasma copper increased and it was high in all trimesters. ${ }^{10}$ Serum copper is significantly increased in GDM patients as compared with healthy pregnant women. ${ }^{11}$

We aimed to see whether the changes in serum zinc, copper together could help in better understanding of the biochemical and metabolic abnormalities in GDM.

\section{Methods}

This case-control study was carried out from July 2013 to June 2014 in the Biochemistry Department of Mymensingh Medical College. Total 172 present subjects were selected by purposive sampling technique from the outpatient department of Obstetrics and Gynaecology and department of Endocrinology in Mymensingh Medical College Hospital. Diagnosed GDM ( $\mathrm{n}=86)$ was in the case group and it was done on the basis of OGTT by WHO criteria 2013. ${ }^{12}$ Case group was subgrouped into Gr-Ia (43) and Gr-Ib (43) at second and third trimester respectively. The normoglycemic pregnant women at second and third trimester were taken as control group $(\mathrm{n}=86)$ and subgrouped as Gr-IIa $(n=43)$ and Gr-IIb $(n=43)$ respectively. All those pregnant women with the previous history of diabetes, hypertension and other endocrine disorders were excluded from this study. This study protocol was approved by the institutional review committee of Mymensingh Medical College. Data were collected through a preformed data collection sheet (questionnaire). The variables were age, education, occupation, socioeconomic status, residential address, dietary habit, height, weight, family history of diabetes, previous pregnancy history and previous history of GDM. Written informed consent was obtained from all the participants of the study groups prior to their enrolment into this study. Blood samples, from pregnant women, which was drawn for OGTT during screening for GDM was used for this study. In this study serum glucose was determined by Enzymatic method with GOD-PAP serum, zinc was determined by colorimetric method with 2-(5-Brom-2-pyridylazo)-5-[-N-propyl-N(3-sulfopropyl) amino]-phenol and serum copper was determined by colorimetric method with the 3,5-DiBrPAESA stain. The results were analyzed and values were expressed as mean $\pm \mathrm{SD}$. The level of significance was determined by employing Student's unpaired ' $t$ ' test. Only when the p value was less than 0.05 ; the difference between two groups and subgroups were considered as statistically significant.

\section{Results}

In this study, 58 out of 86 GDM patients had family history of diabetes mellitus. Baseline chack ristics are shown in Table-I. Serum zinc level was significantly decreased in the GDM women in second trimester as Gr-Ia group compared to healthy controls in the second trimester Gr-IIa group and even when compared GDM women in third trimester GIb and healthy controls in the third trimester Gr-IIb group (Table-II). The present study showed that serum copper level was significantly $(p<0.01)$ increased in the GDM women in second trimester as Gr-Ia group compared to healthy controls in the second trimester Gr-IIa group (Table- III). Moreover it was found highly significantly $(\mathrm{p}<0.001)$ increased when compared GDM women in third trimester GIb and healthy controls in the third trimester Gr-IIb group.

Table I. Clinical and Biochemical Characteristics of the study subjects

\begin{tabular}{lccc}
\hline Variables & $\begin{array}{c}\text { Mean } \pm \text { SD } \\
\text { (cases) }\end{array}$ & $\begin{array}{c}\text { Mean } \pm \text { SD } \\
\text { (controls) }\end{array}$ & $\begin{array}{c}\text { P } \\
\text { value }\end{array}$ \\
\hline Age $($ years $)$ & $28.6 \pm 3.23$ & $27.3 \pm 3.13$ & 0.778 \\
BMI $\left(\mathrm{kg} / \mathrm{m}^{2}\right)$ & $26.4 \pm 1.49$ & $26.3 \pm 1.27$ & 0.774 \\
Zinc $(\mu \mathrm{g} / \mathrm{dl})$ & $45.40 \pm 4.776$ & $67.44 \pm 8.444$ & 0.001 \\
Copper $(\mu \mathrm{g} / \mathrm{dl})$ & $237.00 \pm 10.019$ & $234.60 \pm 4.190$ & 0.042 \\
\hline
\end{tabular}

(Student's $t$ test was used to analyse the data between groups) 
Table II. Mean serum zinc levels comparing between different subgroups $(\mathrm{n}=43)$

\begin{tabular}{llllr}
\hline Variables & Group & Trimester & Mean $\pm \operatorname{SD}(\mu \mathrm{g} / \mathrm{dl})$ & P value \\
\hline GDM & Case $(\mathrm{Gr}-\mathrm{Ia})$ & Second trimester & $43.93 \pm 75.479$ & 0.001 \\
Normal pregnancy & Control (Gr- IIa) & Second trimester & $67.30 \pm 7.812$ & \\
GDM & Case $(\mathrm{Gr}-\mathrm{Ib})$ & Third trimester & $46.86 \pm 3.421$ & 0.001 \\
Normal pregnancy & Control (Gr-IIb) & Third trimester & $67.58 \pm 9.124$ & \\
\hline
\end{tabular}

(Student's - $\mathrm{t}$ test was used to analyses the data between groups)

Table III. Mean serum copper levels comparing between different subgroups $(\mathrm{n}=43)$

\begin{tabular}{llllr}
\hline Variables & Group & Trimester & Mean $\pm \mathrm{SD}(\mu \mathrm{g} / \mathrm{dl})$ & P value \\
\hline GDM & Case $(\mathrm{Gr}-\mathrm{Ia})$ & Second trimester & $230.09 \pm 7.618$ & 0.002 \\
Normal pregnancy & Control (Gr- IIa) & Second trimester & $234.33 \pm 3.809$ & \\
GDM & Case $(\mathrm{Gr}-\mathrm{Ib})$ & Third trimester & $243.91 \pm 6.886$ & 0.001 \\
Normal pregnancy & Control ( Gr-IIb) & Third trimester & $234.88 \pm 4.568$ & \\
\hline
\end{tabular}

(Student' $\mathrm{s} t$ test was used to analyses the data between groups)

\section{Discussion}

Zinc and copper are important trace elements in metabolism, growth, development and reproduction. Zinc may be involved in maintaining normal glucose use and reducing the severity of insulin resistance and diabetes. Evidence for increased requirement of zinc during pregnancy has been reported by Al-Saleh et al. ${ }^{10}$ The current study showed highly significant differences in zinc levels between pregnant women with gestational diabetes and control. This finding is similar to previous reports of Bo et al. ${ }^{12}$ and Khadhem. ${ }^{13}$ Zinc exerts a number of indirect antioxidant functions and its deficiency can decrease the response to insulin, possibly by increased oxidative stress damage, apoptosis and inflammation. ${ }^{14}$ Zinc improves insulin signaling, which promotes glucose uptake. The findings of the current study is in contrast to previous reports of Hamdan et al ${ }^{15}$ which showed no significant differences in zinc levels between pregnant women with gestational diabetes and controls.

In this study, the values of copper in GDM subjects were significantly higher $(p<0.05)$ when compared to the control. Our finding is supported by the studies of Yinsong Wang. ${ }^{10}$ Copper in the blood also depends on the time of gestation. The possible causes of the changes are due to the hormonal, metabolic and enzymatic changes in pregnancy. The increase in serum copper may be due to the increase in the inflammatory response especially in copper containing enzyme (ceruloplasmin) ${ }^{16}$. In diabetes mellitus, oxidative stress seems primarily due to both increased plasma free radical concentrations and a sharp reduction in antioxidant defense. Loven et al found that there is no statistically significant difference in serum copper concentrations between healthy pregnant women and women with GDM. ${ }^{17}$

\section{Conclusion}

Analyzing the finding of the present study, significant alteration in serum zinc and copper levels were observed in GDM patients. This study was done within the context of the facilities available to us, it has got some limitations. As the study was case-control the population size was small. Further studies with large number of subjects and with the application of modern sophisticated technology will give a conclusive decision.

Conflict of interest: Nothing to declare.

\section{References}

1. Diagnostic criteria and classification of hyperglycaemia first detected in pregnancy: a World Health Organization Guideline. Diabetes Res Clin Pract. 2014;103:341-63.

2. Buckl Dunne F. on behalf of the DALI Core Investigator Group.. Gestational diabetes mellitus in Europe: ey, BS, Harreiter J. Damm P, Corcoy R. Chico A, Simmons D, Vellinga, A prevalence, current screening practice and barrier to screening. A review. Diabet Med 2012; 844-54 
3. Gokcel A, BagisT, Killicadag EB, Tarim E, Guvener N. Comparison of the criteria for gestational diabetes mellitus by NDDG and Carpenter and Coustan, and the outcomes of pregnancy. J Endocrinol Invest 2002; 25: 357-61.

4. Kim C, Newton KM, Knopp RH. Gestational diabetes and the incidence of type 2 diabetes. Diabetes Care 2002; 25:186268 .

5. Reece EA, Wu YK, Wiznitzer A. Role of free radical in membrane lipid induced congenital malformation. J Soc Gynaecol Invest 1998; 5:178-87.

6. Jovanovic P. Peterson. Vitamin and mineral deficiency which may predispose to glucose intolerance of pregnancy. J. Am, Coll Nutr 1996; 15(1):14-20.

7. Ladipo OA. Nutrition in pregnancy: mineral and vitamin supplements. American Journal of Clinical Nutrition. 2000; 72: $280-90$.

8. Ajose A, Fasubaa B, Anetor JI, Adelekan DA, Makinde NO. Serum zinc and copper concentrations in Nigerian women with normal pregnancy. Niger Postgrad Med J 2011; 8: 16164.

9. Janet CK. Determinants of maternal zinc status during pregnancy. Am J Clin Nutr M 2000; 71 4s-1343s.

10. Al-Saleh E, Nandakumaran M, Al-Harmi J, Sadan T. Al-Enezi H. Maternal-fetal status of copper, iron, molybdenum, selenium, and zinc in obese pregnant women in late gestation. Biol Trace Elem Res 2006; 113:113-23
11. Bo S, Lezo A, Menato G. Gestational hyperglycemia, zinc, selenium, and antioxidant vitamins. Nutrition 2005; 21:18691.

12. Definition and diagnosis of diabetes mellitus and intermediate hyperglycaemia. Geneva: World Health Organization; 2013.

13. Khadhem H. Level of Serum Copper and Zinc in pregnant Women with Gestational Diabetes Mellitus. J Fac Med Bagdad 2005; 17: 287-89.

14. Lima VB, Sampaio Fde A, Bezerra DL, Moita Neto JM, Marreiro Ddo N. Parameters of glycemic control and their relationship with zinc concentrations in blood and with superoxide dismutase enzyme activity in type 2 diabetes patients. Arq Bras Endocrinol Metabol 2011; 55:701-7.

15. Fox P, Mukhopadhyay C, Ehrenwald E. Structure, oxidant activity and cardiovascular mechanisms of human ceruloplasmin. Life Sci 1995; 56:1749-58.

16. Tang X, Shay NF, Zinc has an insulin-like effect on glucose transport mediated by phosphoinositol-3-kinase and Akt in 3T3-L1 fibroblasts and adipocytes. J Nutr 2001; 131: 141420.

17. Loven A, Romem Y, Pelly IZ, Holeberg G and Agam G. 1992. Copper metabolism - a factor in gestational diabetes? Clin Chim Acta. 192; 31:213:51-59

18. Hamdan HZ, Elbashir LM, Hamdan SZ, Elhassan EM, Adam I. Zinc and selenium levels in women with gestational diabetes mellitus at Medani Hospital, Sudan. J Obstet Gynaecol; 2014; 34:567-70. 